\title{
甘肃白水江国家级自然保护区林缘社区饲养犬只 对大熊猫时空节律的影响
}

滕继荣 ${ }^{1}$, 刘兴明 1 , 何礼文 ${ }^{1}$, 王钧亮 ${ }^{1}$, 黄建 ${ }^{2}$, 冯杰 ${ }^{2}$, 王放 ${ }^{3 *}$, 翁悦 $3^{*}$

1. 甘肃白水江国家级自然保护区, 甘肃陇南 756400; 2. 山水自然保护中心, 北京 $100871 ; 3$. 复旦大学生命科学学院生物多样性与生态工 程教育部重点实验室, 上海 200438

摘要: 人类对于野生动物的影响复杂多样, 其中家养动物所产生的干扰往往被忽视, 例如保护区林缘社区饲养的家犬。半散 放的家犬(Canis familiaris)进入野生动物栖息地, 对保护区内以大熊猫(Ailuropoda melanoleuca)为代表的野生动物可能产生重 要影响。为明确家犬进入林区活动范围对大熊猫的影响, 本研究采用红外相机技术和GPS项圈无线追踪技术对甘肃白水江国 家级自然保护区内家犬对大熊猫潜在栖息地范围和时间节律的影响进行研究, 利用MaxEnt模型对家犬活动范围及大熊猫潜 在栖息地进行预测。结果表明: 甘肃白水江国家级自然保护区内大熊猫潜在栖息地面积为 $885.8 \mathrm{~km}^{2}$, 约占保护区总面积的 $48.2 \%$; 家犬活动面积为 $861.2 \mathrm{~km}^{2}$, 约占保护区总面积的 $47.6 \%$ 。家犬活动范围与大熊猫潜在栖息地重叠的面积约占保护区 面积的 $28.2 \%$, 且多集中在低海拔的常绿阔叶林。大熊猫在有家犬出现的位点和没有家犬出现的位点日活动节律具有显著差 异。本研究揭示了家犬进入林区对大熊猫栖息地潜在影响的空间范围, 为保护区开展生物多样性管理提供了关键信息, 对 于其他保护区具有借鉴意义。

关键词: 大熊猫; 家犬; 栖息地; 红外相机; MaxEnt模型

滕继荣, 刘兴明, 何礼文, 王钧亮, 黄建, 冯杰, 王放, 翁悦 (2022) 甘肃白水江国家级自然保护区林缘社区饲养犬只对大熊猫时空节律的影响. 生物 多样性, 30, 21204. doi: 10.17520/biods.2021204.

Teng JR, Liu XM, He LW, Wang JL, Huang J, Feng J, Wang F, Weng Y (2022) The spatio-temporal impact of domestic dogs (Canis familiaris) on giant panda (Ailuropoda melanoleuca) in Baishuijiang National Nature Reserve. Biodiversity Science, 30, 21204. doi: 10.17520/biods.2021204.

\section{The spatio-temporal impact of domestic dogs (Canis familiaris) on giant panda (Ailuropoda melanoleuca) in Baishuijiang National Nature Reserve}

\author{
Jirong Teng ${ }^{1}$, Xingming Liu ${ }^{1}$, Liwen $\mathrm{He}^{1}$, Junliang Wang ${ }^{1}$, Jian Huang ${ }^{2}$, Jie Feng ${ }^{2}$, Fang Wang ${ }^{\left({ }^{*}\right.}{ }^{*}$, Yue Weng ${ }^{\left(13^{*}\right.}$ \\ 1 Baishuijiang National Nature Reserve, Longnan, Gansu 756400 \\ 2 Shan Shui Conservation Center, Beijing 100871 \\ 3 Ministry of Education Key Laboratory for Biodiversity Science and Ecological Engineering, School of Life Sciences, Fudan \\ University, Shanghai 200438
}

\begin{abstract} dogs.

收稿日期: 2021-05-20; 接受日期: 2021-09-30 基金项

目: 国家自然科学基金(31971537)

* 共同通讯作者 Co-authors for correspondence. E-mail: wfang@fudan.edu.cn; 19210700158@fudan.edu.cn
\end{abstract}

Aims: Anthropogenic interferences have various forms such as domestic animals, in which many have significant negative impacts but are consistently ignored in conservation planning. Most domestic dogs in Baishuijiang live with humans but act as semi-feral dogs since they are allowed to enter forests, including nature reserve, during most of the year (except for planting periods). During these periods of freedom, their home range, activity pattern, and most importantly, interaction with wild animals, remains unknown. To fill this knowledge gap, we chose the giant panda to study the response of wildlife to free-ranging dogs due to the spatial overlap of domestic dogs and giant pandas in Baishuijiang National Nature Reserve. Our objective was to: (1) track the extent of dog movements in and around nature reserve, and (2) evaluate the percent area of nature reserve where giant panda may come in contact with domestic 
Methods: To understand the extent of the impact free-range domestic dogs have on giant pandas, we used infrared cameras and GPS collars to study the repercussions of domestic dogs entering nature reserve and affecting the wildlife. We also constructed a MaxEnt model to estimate the spatial overlap of domestic dogs and giant pandas in Baishuijiang National Nature Reserve.

Results: The suitable habitat for giant pandas in nature reserve is $885.8 \mathrm{~km}^{2}$, around $48.2 \%$ of the overall area, while the area of domestic dog distribution is $861.2 \mathrm{~km}^{2}$, or $47.6 \%$ of the nature reserve. The overlap between domestic dogs and giant panda habitat is $28.2 \%$ of the entire nature reserve. There are significant differences in activity rhythms of giant pandas between sites with and without dog detection.

Conclusion: These results indicate the extent to which the trespassing of domestic dogs has affected giant pandas. The negative impact of residential areas could result in a combination of human activities (e.g., poaching, farming, livestock grazing, automobile traffic), requiring distinctive solutions to eliminate. We suggest any major residents close to nature reserves should restrain dogs at residences. Taking account for the effects of dog's movements and habitat use in the nature reserve is essential for a comprehensive conservation framework.

Key words: giant panda; domestic dog; habitat; camera trap; MaxEnt model

随着人口的增长, 许多家养动物被有意或无意 地引入到各种生态系统中(Hughes \& MacDonald, 2013)。家犬(Canis familiaris)是世界上最具破坏性 的入侵物种之一(Rong et al, 2019), 它是一种杂食 性动物, 从野生动物和腐肉到人类供给的食物都可 以成为家犬的食物(Doherty et al, 2016)。一部分家犬 和人类一起生活但是处于散放状态, 由于有稳定的 食物来源, 家犬的竞争力增强, 因此家犬对于野生 动物的干扰会因此被进一步放大(Vanak \& Gompper, 2009)。研究表明, 家犬通过捕食、竞争等方式干扰 当地野生动物, 对当地的脊椎动物群落具有显著的 负面影响(Young et al, 2011)。有研究表明在野生动 物与家犬共存的地区, 野生动物会避开家犬(Meek, 1999), 例如巴西草原上的鬃狼 (Chrysocyon brachyurus)会避开家犬所在的地区(Lacerda et al, 2009); 智利南部的家犬会影响南美灰狐(Lycalopex griseus) 对栖息地的选择 (Silva-Rodríguez et al, 2010)。同时, 家犬可能通过容易被忽视的方式影响 野生动物, 如狂犬病和犬瘟热病毒的传播(Gehrt et al，2013)。典型的例子是2014年陕西省林业科学院 秦岭大熊猫繁育研究中心(原陕西省珍稀野生动物 抢救饲养研究中心) 圈养的大熊猫 (Ailuropoda melanoleuca) 突发犬瘟热死亡(冯娜, 2017)。虽然家 犬在保护区周围很常见, 但关于它们可能引起的保 护问题的规模和范围的相关研究却很少, 因此了解 家犬对野生动物的影响范围和方式显得尤为重要 (Parsons et al, 2016)。

大熊猫是全球物种保护的旗舰种, 中国生物多 样性保护的伞护种(魏辅文, 2016)。全国第四次大熊
猫调查证实了野生大熊猫种群的恢复, 大熊猫在 IUCN物种红色名录中的级别已经从濒危(EN) 降为 易危(VU) (He et al, 2019)。尽管目前的保护工作取 得了巨大的成就，但中国大熊猫仍然面临长远的生 存挑战, 根本原因是人类不同形式的干扰(Liu et al, 2017)。目前MaxEnt模型多用来量化不同环境因子 对物种栖息地的影响, 其作为评价物种栖息地生境 质量中最常用的模型被广泛运用于各种科学研究, 从两栖类到大型兽类, 从昆虫到植被, 在多物种多 尺度范围内都取得了较好的预测结果(Cao et al, 2013)。红外相机技术作为一种非侵入性采样技术, 可以日夜连续监测, 并且对所研究动物无损伤还能 最大限度地减少对被监测动物造成的压力(Tobler et al，2008), 还可以提供长期和不间断的样本数据, 近年来在野生动物空间分布方面应用广泛( McShea et al, 2016), 因此通过红外相机技术可以探测大熊 猫的分布和栖息地选择, 为大熊猫的保护工作提供 科学支持。

人为干扰对大熊猫及其栖息地的影响是一个 严重的问题, 已引起广泛关注和研究 (Jin et al, 2017)。这一问题的规模和紧迫性, 可能会随着人口 增长而更加严重(White \& Ward, 2010)。目前对于人 类干扰的研究, 多集中于放牧、挖药、打猎等直接 干扰，而家犬是由于人类社会经济活动而被带入保 护区内, 其作为人类活动影响大熊猫栖息地的潜在 途径目前研究较少(张晋东, 2017), 影响程度尚不 明确(Kang, 2021)。本研究采用红外相机技术和GPS 无线追踪技术对甘肃白水江国家级自然保护区(以 下简称白水江保护区) 大熊猫和家犬分布进行研究, 
其主要目的为: (1)探究家犬在保护区及其周围的活 动范围, 评估其活动范围在保护区的占比; (2)探究 保护区内野生大熊猫栖息地分布; (3)计算家犬活动 范围与大熊猫栖息地的重叠比例, 评估家犬对野生 大熊猫产生影响的程度和区域, 以期为大熊猫的保 护提供有效指导, 提高保护措施的有效性。

\section{1 研究区域概况}

白水江保护区 $\left(104^{\circ} 16^{\prime}-105^{\circ} 25^{\prime}\right.$ E， 32 $36^{\circ}-$ $\left.33^{\circ} 00^{\prime} \mathrm{N}\right)$ 位于甘肃省南部, 包括白马河保护站、丹 堡河保护站、刘家坪保护站、让水河保护站、碧口 保护站、红土河保护站和大熊猫驯养繁殖中心 (Wang et al, 2021)。保护区总面积 $1,837.99 \mathrm{~km}^{2}$, 其 中核心区面积 $901.58 \mathrm{~km}^{2}$, 缓冲区 $261.32 \mathrm{~km}^{2}$, 实验 区675.09 $\mathrm{km}^{2}$ 。该地区年均气温 $15.6^{\circ} \mathrm{C}$, 年均降水量 $932.5 \mathrm{~mm}$, 无霜期约 276 天(Rong et al, 2019)。

\section{2 研究方法}

\section{1 数据来源与处理}

\subsection{1 红外相机数据}

大熊猫位点数据主要来自白水江保护区所安 放的红外相机调查数据和全国第四次大熊猫调查 数据。在白水江保护区2017-2020年所进行的红外 相机调查中, 共布设有 170 个红外相机位点, 其中 有64个位点具有大熊猫探测记录。对于红外相机安 装位置选择, 本研究将保护区划分为 $2 \mathrm{~km} \times 2 \mathrm{~km}$ 的 网格，相机布设人员呈“几”或“V”型沿山脊或河道 前进, 采用可变宽样带调查法进行调查, 选取合适 区域安放相机。相机安放原则: 原则上 1 个网格内安 装1台相机; 优先选择兽径、水源地、盐井位置; 安 放高度结合实际情况进行调整; 避免相机前有大石 块或流水, 避免正对大量随风而动的枝叶, 以免引 起相机误触。红外相机设置为每次触发后连续拍摄 3 张照片和 1 段 $30 \mathrm{~s}$ 的视频, 灵敏度设置为“中”, 开 启时间翟, 每台相机配备 1 张 $16 \mathrm{G}$ 或 $32 \mathrm{G}$ 的存储卡 和 8 节南孚 5 号电池, 不设置引诱剂, 不进行隐蔽, 安装完毕后记录相机编号和点位。

\subsubsection{GPS无线追踪项圈数据}

本研究将GPS无线追踪技术用于家犬中, 主要 选择自由散养的家犬, 使用穿戴式GPS项圈(环球 信士HQAN40S野生动物跟踪器), 该项圈重 $220 \mathrm{~g}$,
约占犬体重的 $0.5 \%$, 符合动物遥测技术中跟踪器重 量不超过体重3\%-5\%的经验值(D'Eon \& Delparte, 2005)。自2020年1月至2021年1月对白水江保护区林 缘社区家犬进行项圈佩戴, 选择自由放养并且有可 能进入到保护区内部的家犬, 共佩戴 18 个GPS项圈: 白马河 2 个、丹堡河 4 个、刘家坪 4 个、让水河 4 个、 碧口 4 个。项圈每 $1 \mathrm{~h}$ 记录 1 次, 包含所在位置的经纬 度、海拔、温度、航向、速度等信息(Frair et al, 2004)。 通过项圈的太阳能板对项圈进行供电, 保证工作的 连续性, 家犬所记录的 GPS 位点信息会返回至 Tracker Client软件中, 家犬的出现位点数据主要源于 给家犬所佩戴的GPS项圈, 数据回收后对其进行初 步处理, 剔除信号较弱的位点。

\section{2 数据分析}

\subsection{1 大熊猫和家犬潜在栖息地和活动范围分析}

使用 MaxEnt模型(MaxEnt 3.3.3, https://www. cs.princeton)计算家犬进入林区的活动范围和大熊 猫潜在的栖息地分布范围。对于家犬活动的GPS位 点数据, 我们使用 $2 \mathrm{~h}$ 的间隔进行数据稀释, 并排除 家犬所在居民点的位置 (Anderson \& Gonzalez, 2011)。参考之前的研究, 我们选用海拔、坡度、距 居民点的距离、距道路的距离、距河流的距离、植 被类型作为模型的环境变量。其中海拔、居民点、 道路、河流图层来自于白水江保护区管理局, 并利 用ArcGIS 10.8版本中的“near’工具计算出距离物种 分布位点的欧几里得距离。所有的环境变量均转换 成ASCII格式, 在MaxEnt模型中构建家犬和大熊猫 分布模型, 在构建家犬分布模型后, 我们将家犬活 动范围作为变量带入大熊猫栖息地模型的构建中。 采用受试者工作特征曲线 (receiver operating characteristic curve, ROC) 及其曲线下面积 (area under curve, AUC) 对模型准确性进行检验(Elith et al, 2010)。用平均训练灵敏度和特异性阈值 (equal training sensitivity and specificity threshold) 将 MaxEnt输出的连续分布概率重新分类为分布和未 分布, 并在ArcGIS中利用 'overlap'工具将家犬和大 熊猫的分布范围进行叠加, 计算家犬对大熊猫的影 响范围和保护区占比。

\subsection{2 大熊猫和家犬的重叠日活动节律分析}

根据红外相机拍摄的照片, 我们提取出大熊猫 和家犬出现的时间。采用非参数核密度估计法 
(non-parametric circular kernel-density method)计算 家犬的出现是否会影响大熊猫的日活动节律。将数 据分为两种类型, 有家犬探测的位点和没有家犬探 测的位点。然后比较大熊猫在两种条件下的活动模 式是否有显著差异。使用重叠指数 $(\Delta)$, 范围从 0 (无 重叠)到1 (完全重叠), 来衡量当家犬有被探测或没 有被探测时大熊猫活动的相似性(Linkie \& Ridout, 2011)。随机运行 1,000 次后得到重叠指数和零分布的 标准误差，该分析在R 3.5.3程序中的“overlap”包进 行(Fiske \& Chandler, 2011)。

\section{3 结果}

\section{1 大熊猫潜在栖息地分布结果}

白水江自然保护区探测到大熊猫的位点如图1 所示。根据MaxEnt模型验证结果(AUC = 0.939)可知, 模型拟合程度良好, 预测精度高。MaxEnt模型对大 熊猫潜在栖息地分布范围的计算结果表明, 白水江 保护区内大熊猫栖息地面积为 $885.8 \mathrm{~km}^{2}$, 约占保 护区总面积的 $48.2 \%$ 。大熊猫主要分布在保护区的 核心区，远离林缘居民区(图2)。参与模型运行的环 境变量权重显示, 影响大熊猫分布的主要环境因子 为海拔 (贡献值 $=36.1 \%$ ), 其次为距居民点距离 (贡 献值 $=26.8 \%)$, 家犬是否出现对大熊猫栖息地有 一定潜在的影响(贡献值 $=12.1 \%$ ), 坡度对大熊猫 栖息地选择影响较小(贡献值 $=0.8 \%$ )。

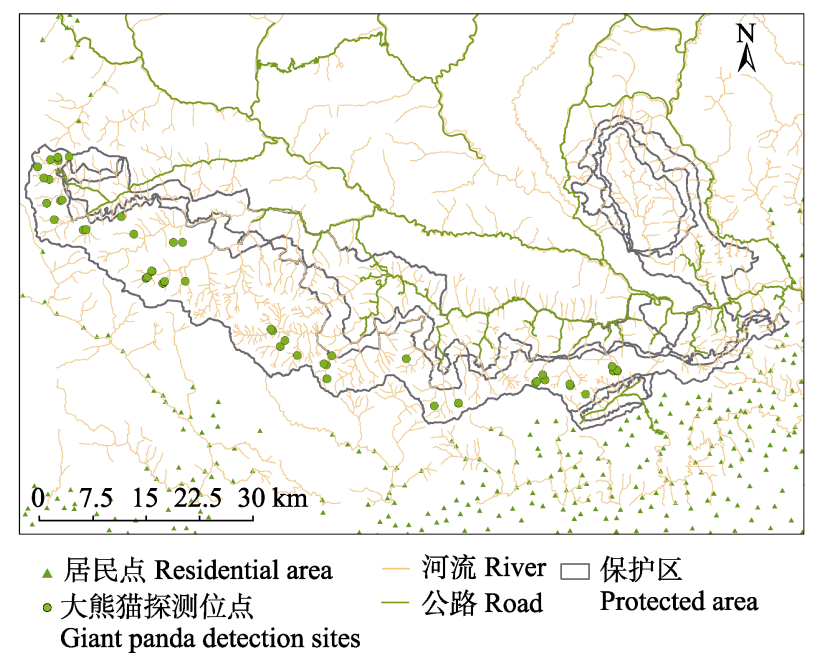

图1 甘肃白水江国家级自然保护区大熊猫探测位点分布图 Fig. 1 Distribution of giant panda detection sites in Baishuijiang National Nature Reserve

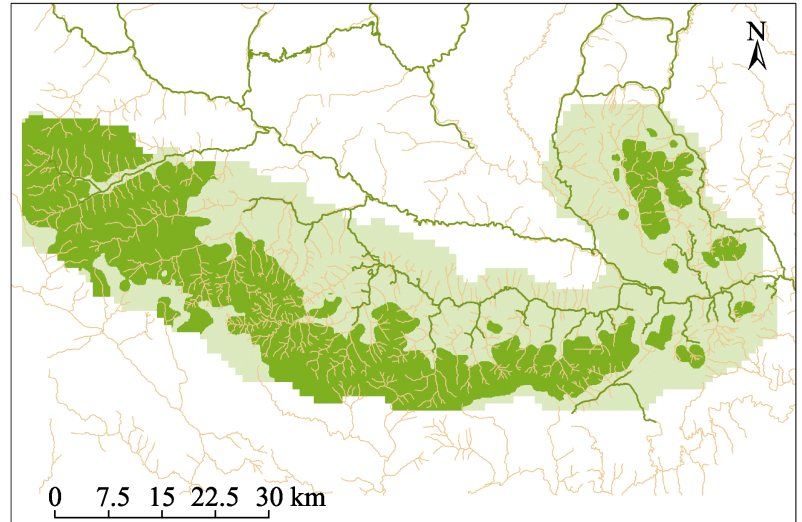

一河流 River $\square$ 大熊猫栖息地

一 公路 Road Potential habitat of giant pandas

图2 基于红外相机位点数据计算出的白水江国家级自然保 护区大熊猫栖息地范围

Fig. 2 The estimated habitat range of giant panda across Baishuijiang National Nature Reserve based on the camera stations

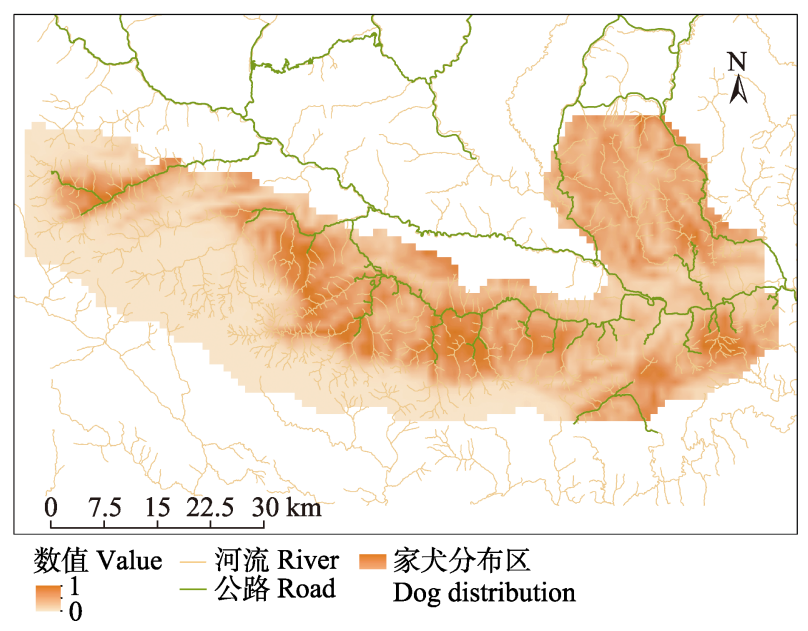

图3 基于18只家犬的GPS项圈数据计算的白水江国家级自 然保护区的家犬活动范围

Fig. 3 The estimated distribution of dogs across Baishuijiang National Nature Reserve based on the tracking movements of 18 dogs

\section{2 家犬活动范围估计}

MaxEnt模型的AUC值为 0.874 , 表明模型拟合 程度良好, 结果准确度较高。模型输出结果表明白 水江保护区内家犬的活动范围为 $861.2 \mathrm{~km}^{2}$, 约占 保护区总面积的 $47.6 \%$ 。活动范围主要集中在保护 区外部，距离道路和居民点较近的区域(图3)。影响 家犬活动范围的主要因子为距离道路的距离(贡献 值 $=56 \%$ ), 其次为坡度 (贡献值 $=20.3 \%$ ), 森林类 型对家犬活动范围的影响较小 (贡献值 $=0.6 \%$ )。 


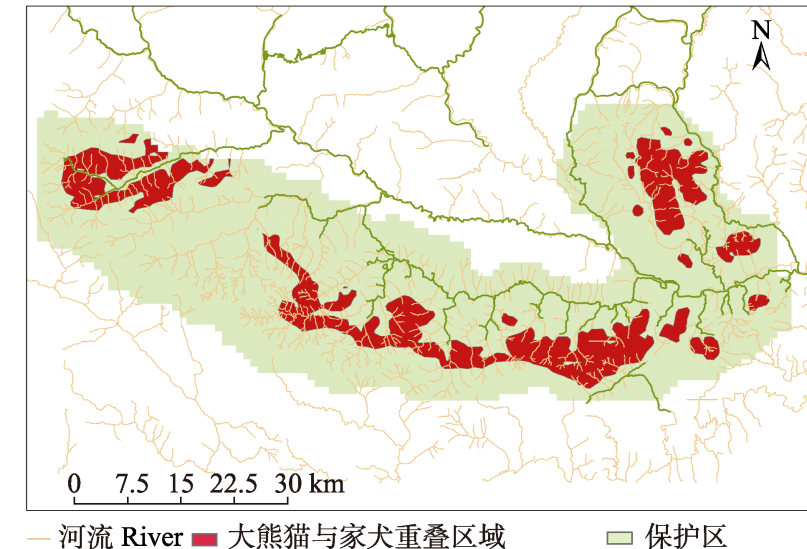

河流 River $\square$ 大熊猫与家犬重叠区域 $\square$ 保护区

一公路 Road The overlap of giant panda and dog Protected area

图4 白水江国家级自然保护区家犬活动范围与大熊猫栖息 地重叠区域

Fig. 4 The overlap between dogs' home range and giant pandas' distributions in Baishuijiang National Nature Reserve

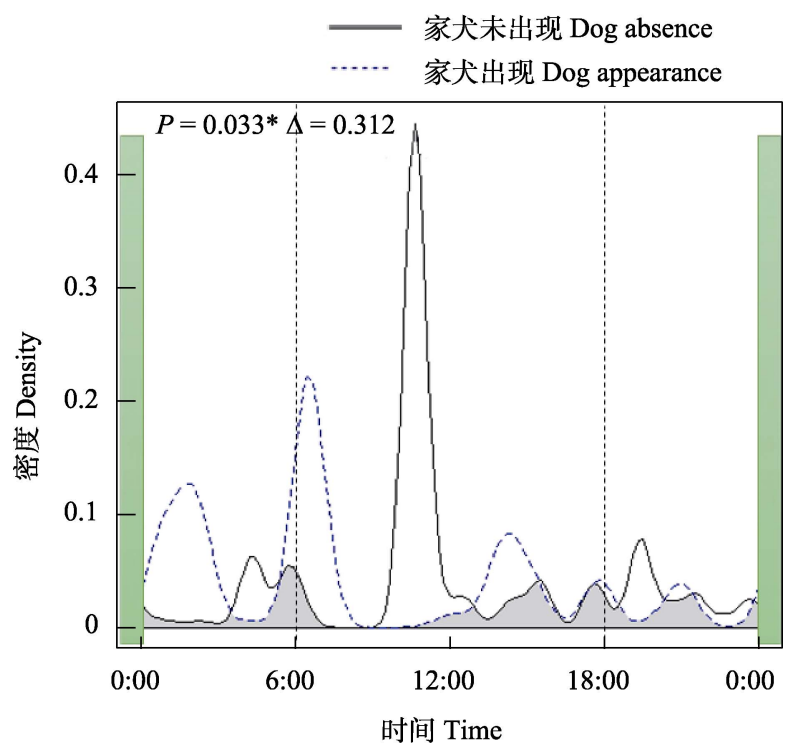

图5 大熊猫在无家犬探测点(黑色实线)和有家犬探测点(蓝 色虚线)的日节律活动。P $<0.05$ 表明有显著性差异。

Fig. 5 Species' daily activity between sites which dog absence (black continuous curves) and dog appearance (blue curves). Significant relationships $(P<0.05)$ are indicated by asterisks.

\section{3 大熊猫和家犬活动范围的重叠}

将大熊猫栖息地与家犬活动范围在ArcGIS中 利用工具“overlap'进行叠加, 结果表明(图4), 家犬 活动范围与大熊猫潜在栖息地重叠部分位于保护 区内部, 其重叠区域面积为 $517.8 \mathrm{~km}^{2}$, 约占大熊猫 潜在栖息地面积的 $58.4 \%$ ，占保护区面积的 $28.2 \%$ 。

\section{4 家犬对大熊猫日活动节律的影响}

在没有家犬活动的位点, 大熊猫的日活动主要
集中于10:00-12:00 (图5)。在有家犬活动的位点, 大 熊猫的日活动主要集中于 $6: 00-8: 00$, 其重叠指数 $(\Delta)$ 为 0.312 , 并且两者之间的 $P$ 值为 0.033 , 表明两 种条件下大熊猫日活动节律有显著性差异。

\section{4 讨论}

本研究以白水江保护区家犬对大熊猫的干扰 为研究目标, 探究家犬进林区活动对大熊猫栖息地 的影响范围以及家犬对大熊猫日活动节律的影响。 研究结果表明家犬在保护区的活动范围与大熊猫 潜在栖息地重叠的面积约占保护区面积的 $28.2 \%$, 模型预测的AUC值都超过 0.87 , 证明模型预测结果 可信，表明保护区周边社区散养的家犬对保护区内 大熊猫从时间和空间上都存在潜在的威胁。

我们对大熊猫潜在栖息地的研究结果表明, 海 拔和居民点是影响大熊猫栖息地的主要因素。保护 区内道路建设可能会改变大熊猫栖息地的适宜性 和格局(He et al，2019), 还可能会造成环境污染 (Zhao et al, 2019), 减少附近森林覆盖(Zhang et al, 2018)，限制道路周围竹子生长等(Zhao et al, 2019)。 从大熊猫栖息地分布范围来看, 约占整个白水江保 护区面积的 $48.2 \%$ 。全国第四次大熊猫调查数据显 示白水江保护区分布有大熊猫 110 只，占甘肃省野 生大熊猫总数的 $83.3 \%$ 。大熊猫主要分布于保护区 的中高海拔和距居民点较远的区域, 这也和之前的 研究结果基本一致(龚明昊等, 2015)。在本研究中我 们发现大熊猫分布对坡度、河流等环境因子不敏感， 我们的研究进一步揭示了影响大熊猫栖息地分布 的环境因素, 考虑到多因素的影响对建立一个全面 的保护框架至关重要。

人类活动对大熊猫栖息地的影响可能是由于 耕作、放牧、交通、噪音或其他原因造成的，家犬 作为人类活动对于大熊猫栖息地干扰的一种复杂 方式, 在过去的研究中常常被忽视。在计算大熊猫 潜在栖息地分布时我们将家犬作为环境变量带入 到模型中, 发现家犬在其中的贡献值为 $12.1 \%$, 说 明家犬对保护区内大熊猫栖息地也有一定的影响。 本研究中的家犬主要是由保护区内林缘社区居民 饲养, 尽管研究结果显示家犬活动区域主要围绕居 民点和附近道路, 但二者活动重叠区域仍然占到大 熊猫栖息地面积的 $58.4 \%$, 比例超过一半。大量研究 
发现, 即使家犬的密度很低, 也会对野生动物的空 间分布造成影响，在家犬与野生动物共存的区域, 野生动物可能趋向于回避家犬的活动范围(Elith et al，2010)。之前的研究也证实大熊猫倾向于回避使 用家养动物干扰过的栖息地(魏辅文, 2016)。在本研 究中, 影响大熊猫栖息地的主要因素为海拔, 其次 是距道路的距离。由于人类对于家犬的饲养作用, 导致有源源不断的食物供给, 因此很可能死亡率较 低, 家犬数量可能会持续增加, 导致家犬对于大熊 猫的影响作用可能被进一步放大。之前的研究表明 同域野生哺乳动物可能采取不同的策略来应对家 犬，除了改变其他物种对栖息地的利用, 野生动物 还可能改变日常活动, 以维持它们的活动范围 (Silva-Rodríguez et al, 2010)。在本研究中大熊猫在 探测到家犬的位点和没有探测到家犬的位点日活 动节律具有显著差异, 这种差异可能是由于家犬在 保护区内的出现对大熊猫产生了负面影响, 而动物 活动节律的形成是动物对环境和各种因素变化的 综合适应，也受到各种内外因素的影响。

了解物种之间的相互作用及其影响范围, 有助 于解释生物多样性的维持, 帮助管理者做出合理的 保护决策(Kennedy et al, 2018)。我们的研究表明, 白水江保护区内家犬进林活动范围和大熊猫栖息 地有显著的重叠, 但保护区内部重叠面积较少。从 整体和长远物种保护的角度来看, 白水江保护区内 常住人口数量庞大, 饲养家犬数量较多, 因此家犬 对于保护区内以大熊猫为代表的野生动物及栖息 地的影响不容忽视。有研究表明, 制定家犬管理规 则有助于控制家犬的行为, 并能降低家犬对于野生 动物的影响(Paschoal et al, 2016)。最显著的解决方 案是尝试将家犬从该保护区内移走, 但由于社区经 济文化等原因很难实行。然而, 有研究表明, 增加 拴绳率的管理可以明显减少家犬干扰对野生动物 健康的间接影响(Weston et al, 2014)。因此, 本研究 建议: (1)这些自然保护区建立更全面、更有效的犬 类管理制度。(2)对靠近大熊猫栖息地的家养犬只拴 养并持续开展犬只的免疫, 严格管理家养犬的品 种、控制数量增长等。(3)通过全面开展环境教育活 动, 提高人们对饲养、免疫和绝育犬只的责任感。

\section{ORCID}

王放 (iD) https://orcid.org/0000-0002-3922-5851
翁悦 (D) https://orcid.org/0000-0002-1926-700X

\section{参考文献}

Anderson RP, Gonzalez IJ (2011) Species-specific tuning increases robustness to sampling bias in models of species distributions: An implementation with Maxent. Ecological Modelling, 222, 2796-2811.

Cao Y, DeWalt RE, Robinson JL, Tweddale T, Hinz L, Pessino M (2013) Using Maxent to model the historic distributions of stonefly species in Illinois streams: The effects of regularization and threshold selections. Ecological Modelling, 259, 30-39.

D'Eon RG, Delparte D (2005) Effects of radio-collar position and orientation on GPS radio-collar performance, and the implications of PDOP in data screening. Journal of Applied Ecology, 42, 383-388.

Doherty TS, Glen AS, Nimmo DG, Ritchie EG, Dickman CR (2016) Invasive predators and global biodiversity loss. Proceedings of the National Academy of Sciences, USA, 113, 11261-11265.

Elith J, Graham CH, Anderson RP, Dudík M, Ferrier S, Guisan A, Hijmans RJ, Huettmann F, Leathwick JR, Lehmann A (2010) Novel methods improve prediction of species' distributions from occurrence data. Ecography, 29, 129-151.

Feng N (2017) Epidemiological Investigation and Experimental Immune Study on Canine Distemper in Giant Panda. PhD dissertation, Jilin Agricultural University, Changchun. (in Chinese with English abstract) [冯娜 (2017) 大熊猫犬瘟 热流行病学调查与实验免疫研究. 博士学位论文, 吉林 农业大学, 长春.]

Fiske I, Chandler R (2011) Unmarked: An R package for fitting hierarchical models of wildlife occurrence and abundance. Journal of Statistical Software, 43, 1-23.

Frair JL, Nielsen SE, Merrill EH, Lele SR, Boyce MS, Munro RHM, Stenhouse GB, Beyer HL (2004) Removing GPS collar bias in habitat selection studies. Journal of Applied Ecology, 41, 201-212.

Gehrt SD, Wilson EC, Brown JL, Anchor C (2013) Population ecology of free-roaming cats and interference competition by coyotes in urban parks. PLoS ONE, 8, e75718.

Gong MH, Ouyang ZY, Xu WH, Song YL, Dai B (2015) The location of wildlife corridors under the impact of road disturbance: Case study of a giant panda conservation corridor. Acta Ecologica Sinica, 35, 3447-3453. (in Chinese with English abstract) [龚明昊, 欧阳志云, 徐卫华, 宋延 龄, 戴波 (2015) 道路影响下野生动物廊道的选址以大熊猫保护廊道为例. 生态学报, 35, 3447-3453.]

He K, Dai Q, Gu XH, Zhang ZJ, Zhou J, Qi DW, Gu XD, Yang XY, Zhang W, Yang B, Yang ZS (2019) Effects of roads on giant panda distribution: A mountain range scale evaluation. Scientific Reports, 9, 1110.

Hughes J, MacDonald DW (2013) A review of the interactions 
between free-roaming domestic dogs and wildlife. Biological Conservation, 157, 341-351.

Jin YP, Zhang XK, Ma YS, Qiao YC, Liu XB, Zhao KH, Zhang CL, Lin DG, Fu XL, Xu XR, Wang YW, Wang HN (2017) Canine distemper viral infection threatens the giant panda population in China. Oncotarget, 8, 113910-113919.

Kang DW (2021) A review of the impacts of four identified major human disturbances on the habitat and habitat use of wild giant pandas from 2015 to 2020 . Science of the total Environment, 763, 142975.

Kennedy B, Brown W, Vernes K, Körtner G, Butler J (2018) Dog and cat interactions in a remote aboriginal community. Animals, 8, 65.

Lacerda ACR, Tomas WM, Marinho-Filho J (2009) Domestic dogs as an edge effect in the Brasília National Park, Brazil: Interactions with native mammals. Animal Conservation, 12, 477-487.

Linkie M, Ridout MS (2011) Assessing tiger-prey interactions in Sumatran rainforests. Journal of Zoology, 284, 224-229.

Liu XH, Wu PF, Shao XM, Songer M, Cai Q, Zhu Y, He XB (2017) Spatiotemporally monitoring forest landscape for giant panda habitat through a high learning-sensitive neural network in Guanyinshan Nature Reserve in the Qinling Mountains, China. Environmental Earth Sciences, 76, 1-12.

McShea WJ, Forrester T, Costello R, He ZH, Kays R (2016) Volunteer-run cameras as distributed sensors for macrosystem mammal research. Landscape Ecology, 31, 55-66.

Meek PD (1999) The movement, roaming behaviour and home range of free-roaming domestic dogs, Canis lupus familiaris, in coastal New South Wales. Wildlife Research, 26, 847-855.

Parsons AW, Bland C, Forrester T, Baker-Whatton MC, Schuttler SG, McShea WJ, Costello R, Kays R (2016) The ecological impact of humans and dogs on wildlife in protected areas in eastern North America. Biological Conservation, 203, 75-88.

Paschoal AMO, Massara RL, Bailey LL, Kendall WL, Doherty PF Jr, Hirsch A, Chiarello AG, Paglia AP (2016) Use of Atlantic Forest protected areas by free-ranging dogs: Estimating abundance and persistence of use. Ecosphere, 7, 115.

Rong ZL, Liu XM, Zhao CY, He LW, Liu JJ, Gao YF, Zang F, Xu HJ, Guo ZX, Mao YH (2019) Evaluating giant panda as a surrogate species for conservation co-occurring species in the Baishuijiang National Nature Reserve. Environmental Science and Pollution Research, 26, 8939-8948.

Silva-Rodríguez EA, Ortega-Solís GR, Jiménez JE (2010) Conservation and ecological implications of the use of space by Chilla foxes and free-ranging dogs in a human-dominated landscape in southern Chile. Austral Ecology, 35, 765-777.
Tobler MW, Carrillo-Percastegui SE, Leite Pitman R, Mares R, Powell G (2008) An evaluation of camera traps for inventorying large- and medium-sized terrestrial rainforest mammals. Animal Conservation, 11, 169-178.

Vanak AT, Gompper ME (2009) Dogs (Canis familiaris) as carnivores: Their role and function in intraguild competition. Mammal Review, 39, 265-283.

Wang H, Liu XM, Zhao CY, Chang YP, Liu YY, Zang F (2021) Spatial-temporal pattern analysis of landscape ecological risk assessment based on land use/land cover change in Baishuijiang National Nature Reserve in Gansu Province, China. Ecological Indicators, 124, 107454.

Wei FW (2016) Research progress in conservation biology of endangered mammals in China. Acta Theriologica Sinica, 36, 255-269. (in Chinese with English abstract) [魏辅文 (2016) 我国濒危哺乳动物保护生物学研究进展. 兽类学 报, 36, 255-269.]

Weston MA, Fitzsimons JA, Wescott G, Miller KK, Ekanayake KB, Schneider T (2014) Bark in the park: A review of domestic dogs in parks. Environmental Management, 54, 373-382.

White PCL, Ward AI (2010) Interdisciplinary approaches for the management of existing and emerging human-wildlife conflicts. Wildlife Research, 37, 623-629.

Young JK, Olson KA, Reading RP, Amgalanbaatar S, Berger J (2011) Is wildlife going to the dogs? Impacts of feral and free-roaming dogs on wildlife populations. BioScience, 61, 125-132.

Zhang JD (2017) The research progress of the effects of human and natural disturbance on giant panda (Ailuropoda melanoleuca). Journal of China West Normal University (Natural Sciences), 38, 227-233. (in Chinese with English abstract) [张晋东 (2017) 人类与自然干扰对大熊猫影响 的研究进展. 西华师范大学学报(自然科学版), 38, 227233.]

Zhang JJ, Jiang F, Li GY, Qin W, Li SQ, Gao HM, Cai ZY, Lin GH, Zhang TZ (2019) Maxent modeling for predicting the spatial distribution of three raptors in the Sanjiangyuan National Park, China. Ecology and Evolution, 9, 6643-6654.

Zhang YK, Wu YJ, Zhang QY, Ran JH, Price M (2018) Distribution of a giant panda population influenced by land cover. The Journal of Wildlife Management, 82, 1199-1209.

Zhao Y, Chen YP, Ellison AM, Liu WG, Chen D (2019) Establish an environmentally sustainable giant panda national park in the Qinling Mountains. Science of the total Environment, 668, 979-987. 